\title{
INTERNAL FRICTION IN F.C.C. ALLOYS DUE TO SOLUTE DRAG ON DISLOCATION-II. EXPERIMENTAL STUDIES ON Al-Si ALLOYS
}

\author{
A. PICHLER, $\dagger$ M. WELLER and E. ARZT
}

Max-Planck-Institut für Metallforschung and Institut für Metallkunde der Universität Stuttgart, D-70174 Stuttgart, Germany

(Received 16 July 1993; in revised form 23 February 1994)

\begin{abstract}
Internal friction (mechanical loss) experiments were carried out on single crystal and polycrystalline Al-Si alloys with incoherent Si particles of varying size, spacing, and as a function of pre-strain. The resulting internal friction spectra are correlated with microstructural investigations. The loss maximum at about $450 \mathrm{~K}(f \sim 1 \mathrm{~Hz})$, especially its "low" activation enthalpy of $H=1.14 \mathrm{eV}$, can be explained by our dislocation model described in the companion paper (Part I), which considers the enhanced transverse diffusivity of solute atoms in the vicinity of the dislocation core. The model is also shown to be in agreement with published data on $\mathrm{Al}-\mathrm{Si}, \mathrm{Al}-\mathrm{Mg}, \mathrm{Cu}-\mathrm{Si}$ and $\mathrm{Cu}-\mathrm{Al}$. It is proposed that in many instances the internal friction maxima, even when characterized by a "low" activation enthalpy, need not be due to grain boundary or particle effects, but may well be explained by core diffusion effects on solute drag acting on dislocations.
\end{abstract}

\section{INTRODUCTION}

Many aluminium alloys with incoherent particles exhibit, regardless of their composition, internal friction (mechanical loss) maxima at temperatures between 400 and $500 \mathrm{~K}$ at about $1 \mathrm{~Hz}$ [1-6]. For explaining the maxima, different mechanisms are proposed and discussed in the literature. Most authors invoke a grain boundary mechanism, in which the particles reduce the sliding distance between the triple nodes and therefore lower the temperature of the grain boundary maximum $[4,6-8]$. The other mechanism $[9,10]$ is the relaxation of stress in the particles by diffusion in the particle-matrix interface.

Some authors report conflicting evidence from which it is not clear which mechanistic interpretation should be favored. For example, Okabe et al. [10] carried out internal friction measurements on both single crystal and polycrystalline Al samples with incoherent $\mathrm{Si}$ particles of different sizes and found similar maxima in both types of specimens. They obtained good agreement between the experimental results and the model of Mori et al. [9]. By contrast, Entwistle et al. [11] found the maximum only in polycrystalline samples of similar $\mathrm{Al}-\mathrm{Si}$ alloys and attributed it to grain boundary sliding. An alternative explanation, which was not considered in either investigation, lies in the possible role of dislocations. For f.c.c. metals containing impurities, mechanisms in which the kinetics of the dislocation motion is

†Present address: VOEST ALPINE Stahl Linz GmbH, Linz, Austria. determined by the concurrent movement of a Cottrell cloud, e.g. as in the model of Schoeck [12, 13], have not been invoked for explaining experiments in the past.

In this paper new internal friction experiments on $\mathrm{Al}-\mathrm{Si}$ alloys with incoherent $\mathrm{Si}$ particles are reported. To illuminate the possible influences of dislocations, grain boundaries, particles, and the interaction between these defects, samples with different microstructures, i.e. grain size, particle size, and dislocation density and arrangement, were produced and investigated. In particular, the behavior of single crystals was compared with that of polycrystals. From a correlation between the different internal friction spectra and the microstructure, as investigated by TEM, conclusions on the mechanisms are drawn. The experimental results are discussed in the light of our recent dislocation model [14], in which the enhanced diffusion of the solute atoms in the dislocation core is considered. It is also shown that the contradiction between the results of Okabe $e t$ al. and Entwistle et al. may thus be resolved and that other experimental results on $\mathrm{Al}$ and $\mathrm{Cu}$ alloys can be explained in a natural way.

\section{EXPERIMENTAL}

\subsection{Preparation and characterization of the Al-Si alloy}

Sample preparation started from $5 \mathrm{~N} \mathrm{Al}(\mathrm{Cu} \sim 0.6$ ppm, $\mathrm{Mg} \sim 0.7 \mathrm{ppm}, \mathrm{Si} \sim 1 \mathrm{ppm}$ ) and $6 \mathrm{~N} \mathrm{Si}$, which were molten and cast in an $\mathrm{Al}_{2} \mathrm{O}_{3}$ crucible under $\mathrm{Ar}$. The castings were swaged from a diameter of 10 to 
Table 1. The Al-Si specimens investigated

\begin{tabular}{lcccccc}
\hline Sample & $f_{\mathrm{V}}(\%)$ & $T(\mathbf{K})$ & $t(\mathrm{~h})$ & $a(\mathrm{~nm})$ & $l_{\mathrm{p}}(\mathrm{nm})$ & $\varphi$ \\
\hline S1 & 0.72 & 553 & 10 & 53 & 452 & Handling \\
S2 & 0.60 & 573 & 10 & 64 & 598 & Handling \\
S3 & 0.54 & 593 & 10 & 73 & 720 & Handling \\
SD & 0.69 & 573 & 10 & 62 & 540 & 0.15 \\
P & 0.52 & 573 & 10 & 58 & 582 & Handling \\
\hline
\end{tabular}

$f_{\sqrt{ }}$-volume fraction of the Si precipitations, $T$-aging temperature, $t$-aging time, $a$-particle size, $l_{\mathrm{p}}$-mean planar particle distance, and $\varphi$-deformation

$4.5 \mathrm{~mm}$. A recrystallization treatment at $T=520^{\circ} \mathrm{C}$ for $3 \mathrm{~min}$ resulted in a grain size of $0.1 \mathrm{~mm}$. Single crystals were produced with the strain-anneal method [15], involving a critical straining of $1.2 \%$ and a travelling velocity of $1 \mathrm{~cm} / \mathrm{h}$ to a hot $(853 \mathrm{~K})$ salt bath. In order to obtain different particle distributions, samples were aged at various temperatures for $10 \mathrm{~h}$. The parameters and the nomenclature of the samples are listed in Table 1. The Si content was determined by wet chemistry, and the volume fraction of $\mathrm{Si}$ particles was calculated under the assumption that $\mathrm{Si}$ is insoluble in Al. From the volume fraction $f_{\mathrm{V}}$ and the mean particle size $a$, the mean planar particle distance $l_{\mathrm{p}}=a \cdot\left(\pi / 6 \cdot f_{\mathrm{v}}\right)^{0.5}$ was obtained (Table 1). To investigate the influence of deformation, a single crystal (SD) was deformed to about $15 \%$ by rolling. Samples with dimensions $4 \times 1 \times 50 \mathrm{~mm}^{3}$ were prepared by spark cutting for the internal friction measurements.

The microstructure was mainly investigated with a JEOL 200CX TEM. After grinding to $100 \mu \mathrm{m}$, the samples were thinned at $T=243 \mathrm{~K}$ and at a voltage of 30-35 V with an electrolyte containing one part $\mathrm{HNO}_{3}$ and two parts $\mathrm{CH}_{3} \mathrm{OH}$. The size of the $\mathrm{Si}$ precipitates was determined from bright field images with the semi-automatic image analyzer "Videoplan". Weak beam images were produced for the study of dislocation structures. The grain size of the polycrystalline sample $P$ was measured in the light microscope by the mean-intercept method, after an etching treatment following Barker [16].

\subsection{Internal friction measurements}

Internal friction (mechanical loss) measurements were carried out with an automatic computercontrolled inverted torsion pendulum [17]. Two types of experiments were performed with this equipment: (i) In the pendulum arrangement different inertias, corresponding to frequencies between 1 and $15 \mathrm{~Hz}$, were used. The internal friction was determined from the logarithmic decrement, $\delta$, of the freely decaying torsional oscillations according to $Q^{-1}=\delta / \pi(1-\delta /$ $2 \pi)$. The measurements were performed with increasing temperature, with a mean heating rate of about $2 \mathrm{~K} / \mathrm{min}$. The maximum shear strain at the surface was between 1 and $12 \times 10^{-5}$. (ii) In another arrangement [18], in which the inertia parts of the pendulum are removed, the loss angle $\varphi \mathrm{h} \simeq Q^{-1}$-i.e. the angle between applied stress and resulting strain-was measured for forced vibrations at constant tempera- ture by variation of the applied frequency $f(f=\omega / 2 \pi)$ between $10^{-4}$ and $40 \mathrm{~Hz}$. The accuracy of $\varphi$ was estimated from repeated measurements as $\Delta \varphi=$ $\pm 2 \div 5 \times 10^{-4} \mathrm{rad}$. The temperature drift during one $\bar{Q}^{-1}(\omega)$ measurements (extending over 8-12 h) was smaller than $1 \mathrm{~K}$. For all experiments a static pressure of $1 \mathrm{mbar} \mathrm{He}$ was maintained inside the pendulum.

The measured loss spectra were decomposed by fitting with Debye maxima including a log-normal distribution of relaxation times [19]. For the $Q^{-1}(T)$ measurements we subtracted a background (increasing with temperature) consisting of a superposition of a linear and exponential term. For the $Q^{-1}(\omega)$ measurements different backgrounds were subtracted at high and low frequencies. The low frequency part was fitted by a simple approximation corresponding to the tail of a Debye maximum with a log-normal distribution, $Q_{\mathrm{BL}}^{-1}=A / \omega^{B}$, where $A$ and $B$ are fit parameters. The high frequency background, resulting mainly from the mechanical parts of the equipment, was fitted by $Q_{\mathrm{BH}}^{-1}=C \cdot \omega+D \cdot \tan (\varphi) \cdot \omega^{2}$, where $C$ and $D$ are fit parameters [20].

From the displacement of the temperature $T_{\mathrm{p}}$ at the maximum (measurements with variable temperature) and the frequency $\omega_{\mathrm{p}} / 2 \pi$ at the maximum (measurements with variable frequency), the activation enthalpy $H$ and the inverse attempt frequency $\tau_{0}$ of the relaxation process were calculated according to

$$
\omega_{p} \cdot \tau_{p}=1=\omega_{p} \cdot \tau_{0} \cdot \exp \left(H / k T_{p}\right) .
$$

\section{RESULTS}

\subsection{Microstructure}

Figure 1 shows a typical microstructure of a single crystal (S2). The dislocation density is for a single crystal surprisingly high, which may be explained by deformation during spark machining. It is remarkable that most of the dislocations are in contact with the particles, which act as firm pinning points. For the deformed single crystal (SD), it is difficult to visualize a representative microstructure as during the first measurement serious alterations take place.

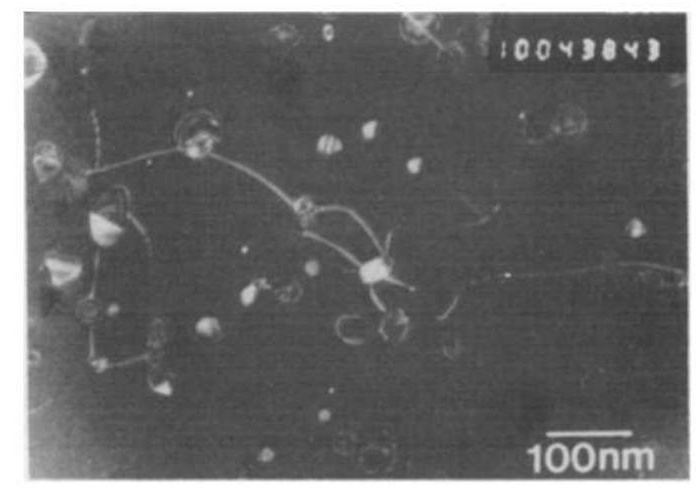

Fig. 1. TEM micrograph of single crystal S2 showing the arrangement of the Si particles and the dislocation structure. (Specimens S1 and S3 have similar microstructure.) 


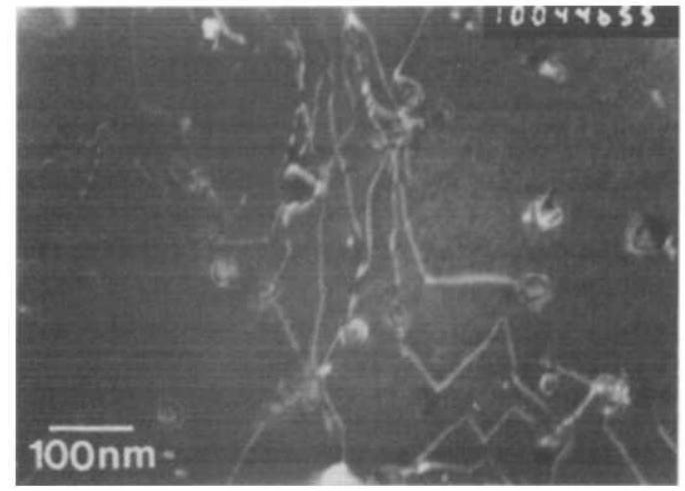

Fig. 2. TEM micrograph showing the dislocation structure in a rolled (strain $\varphi=15 \%$ ) single crystal (SD) after thermal treatment at $450 \mathrm{~K}$ for $10 \mathrm{~min}$.

To simulate the microstructure which is expected at the maximum temperature of the first run, a sample was annealed at $450 \mathrm{~K}$ for $10 \mathrm{~min}$ after deformation. In such a pre-treated single crystal the dislocation density is higher than in the as-grown state by about a factor of 5 (Fig. 2). In some regions the dislocations are arranged in irregular networks. For producing a microstructure typical of the second run of the internal friction measurements, a sample was annealed at $540 \mathrm{~K}$ for $2 \mathrm{~h}$ after deformation. During this treatment the dislocation density decreased significantly (Fig. 3). In particular, the appearance of nearly

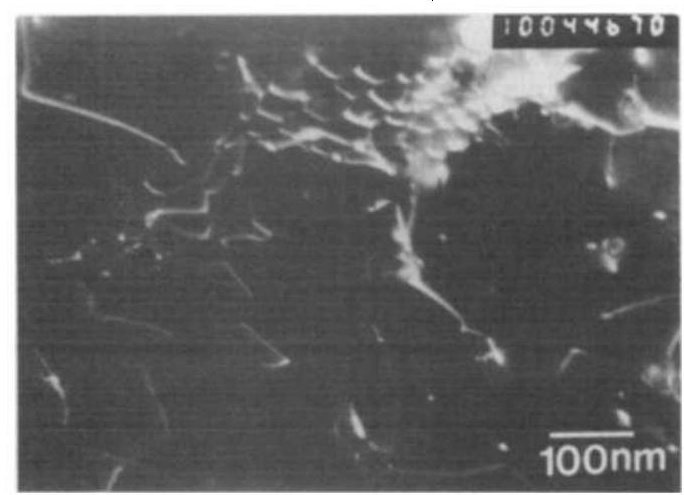

Fig. 3. As in Fig. 2 after thermal treatment at $540 \mathrm{~K}$ for $2 \mathrm{~h}$.

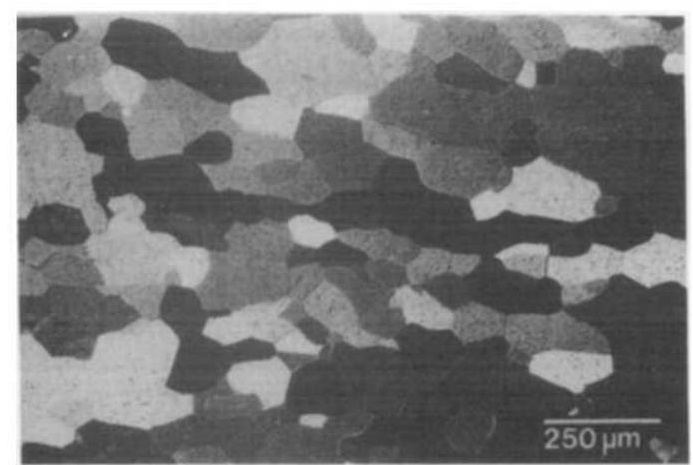

Fig. 4. Light micrograph of the grain structure in sample $P$, etched according to Barker [16].

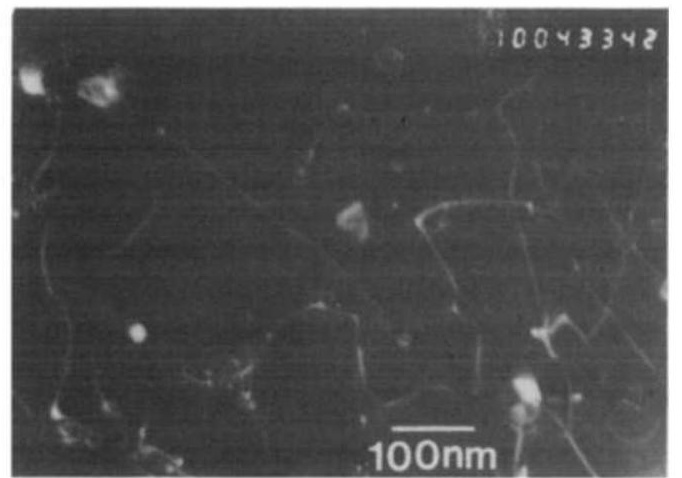

Fig. 5. TEM micrograph of the dislocation structure in the polycrystalline sample $P$.

perfect dislocation networks is observed pointing to strong recovery.

The grain size in the polycrystalline material $P$ is $0.1 \mathrm{~mm}$ (Fig. 4). The arrangement of the dislocations is similar as in $\mathrm{S} 2$; only the dislocation density is $2-5$ times higher (Fig. 5).

\subsection{Internal friction spectra}

As shown in Fig. 6, a loss maximum was detected in the polycrystalline sample $P$ and a single crystal (S2) at nearly the same temperature, but the relaxation strength in $P$ is 5 times higher than in the single crystal. The deformed single crystal (SD) shows a higher maximum at a lower temperature with a more pronounced background. In a second measurement of the same sample, the height of the maximum decreased by about $80 \%$, whereas the background remained unchanged. Additional measurements did not produce further changes in the spectrum.

To investigate the influence of different particle distributions, experiments were conducted on single crystals which had been subjected to different aging treatments (Table 1). For coarser particles (larger particle sizes and mean planar particle distances), the

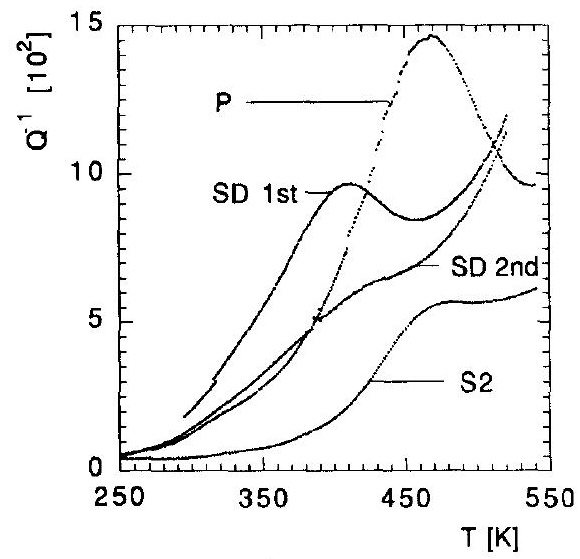

Fig. 6. Internal friction spectra $Q^{-1}(T)$ for the polycrystalline sample (P), an undeformed single crystal (S2) and a deformed single crystal (SD). Measurement frequency about $3.5 \mathrm{~Hz}$. 


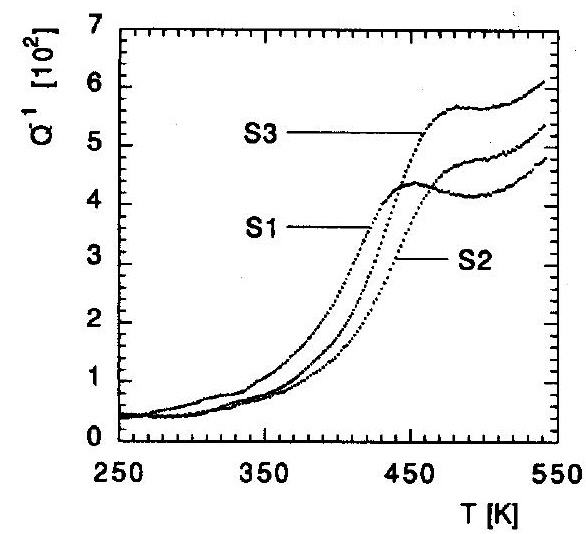

Fig. 7. Internal friction spectra for three single crystals with increasing particle sizes and spacings $(\mathrm{S} 1 \rightarrow \mathrm{S} 2 \rightarrow \mathrm{S} 3)$. The temperatures at the maximum are $458 \mathrm{~K}$ (S1), $463 \mathrm{~K}$ (S2) and $470 \mathrm{~K}$ (S3).

Table 2. The activation enthalpy $H$ and the inverse attempt frequency $\tau_{0}$ for the single crystal $S 2$ and the polycrystalline sample

\begin{tabular}{llcc}
\hline \multicolumn{3}{c}{$\tau_{0}(\mathrm{~s})$} & $H(\mathrm{eV})$ \\
\hline S2 & $T$ variation & $1.6 \times 10^{-15}$ & 1.23 \\
$\mathrm{P}$ & $T$ variation & $2.4 \times 10^{-14}$ & 1.14 \\
& $f$ variation & $2.9 \times 10^{-15}$ & 1.16 \\
\hline
\end{tabular}

maximum shifts to higher temperatures (Fig. 7) while the relaxation strength does not change significantly.

Values of the activation enthalpy $H$ and the inverse attempt frequency $\tau_{0}$ are listed in Table 2. For the polycrystalline sample, the activation enthalpy was determined from measurements with different frequencies $Q^{-1}(f)$ and temperatures $Q^{-1}(T)$ (Fig. 8). For fitting the $Q^{-1}(T)$ measurements the observed asymmetry of the maximum was taken into account by superimposing a small maximum at the low temperature end (Fig. 9). For the single crystal the activation enthalpy was determined only from $Q^{-1}(T)$ measurements.

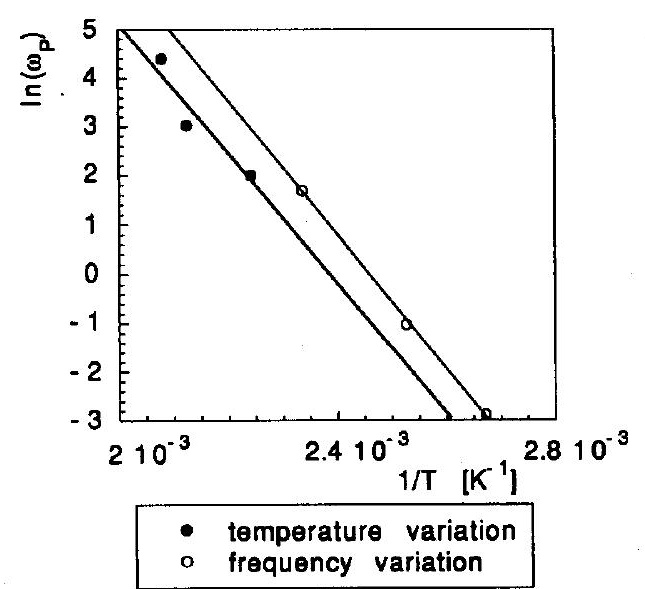

Fig. 8. Arrhenius plots for the internal friction maxima $\left(\ln \omega_{\mathrm{p}}\right.$ vs $\left.1 / T\right)$ obtained for $Q^{-1}(T)$ and $Q^{-1}(\omega)$ measurements $\left(\omega_{\mathrm{p}}\right.$ and $T_{\mathrm{p}}$ denote the angular frequency and the temperature at the maximum, resp.).

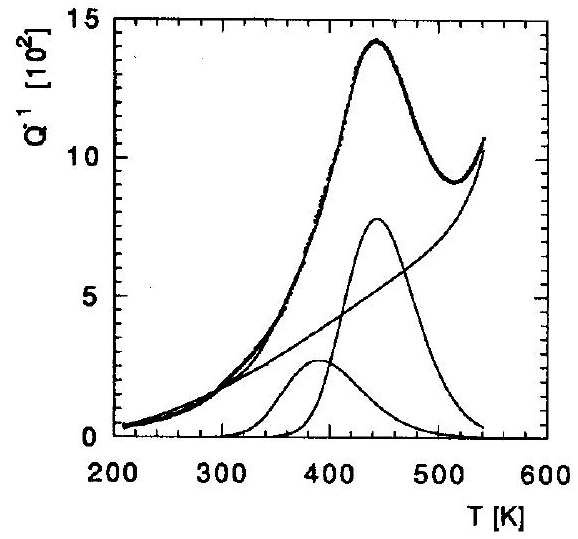

Fig. 9. Decomposition of the internal friction spectrum of the polycrystalline sample $P$. The smaller maximum at lower temperatures compensates the asymmetry of the maximum.

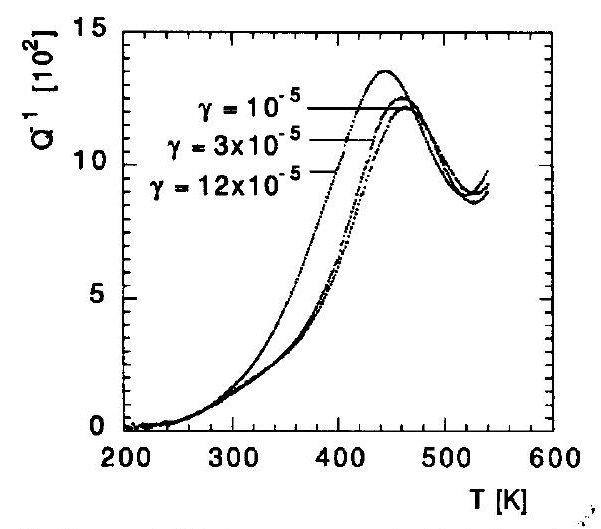

Fig. 10. Internal friction spectra $Q^{-1}(T)$ for the polycrystalline specimen $\mathbf{P}$ for three different shear amplitudes.

The internal friction in specimen $\mathbf{P}$ was measured for various shear strain amplitudes $\gamma$ (Fig. 10). For increasing amplitudes the maximum shifts from $463 \mathrm{~K}\left(\gamma=10^{-5}\right)$ to $444 \mathrm{~K}\left(\gamma=12 \times 10^{-5}\right)$ and the relaxation strength increases by about $20 \%$. From this shift, the activation volume $v_{\mathrm{ex}}$ was estimated using $\left.\tau=\tau_{0} \cdot \exp \left[\left(H-\sigma_{x y} \cdot v_{\mathrm{ex}}\right) / k T\right)\right]$, where $\sigma_{x y}$ is the shear stress $\sigma_{x y}=G \cdot \gamma$ and $G$ the shear modulus. The experimental data can be well fitted with $v_{\text {ex }}=180 \cdot b^{3}$.

\section{DISCUSSION}

\subsection{Possible mechanisms for internal friction}

According to the literature the maximum at $450 \mathrm{~K}$ (for a testing frequency of about $3.5 \mathrm{~Hz}$ ) can be attributed to (i) grain boundary sliding [7, 21, 22], (ii) relaxation of stresses in the particles [9] or (iii) dislocation mechanisms [13, 23-25]. In our experiments the first two may be ruled out for the following reasons: a grain boundary mechanism (i) cannot produce a similar maximum in both the single crystal and the polycrystalline sample, let alone with the same relaxation parameters. For mechanism (ii) the relaxation strength should scale linearly with the volume 
fraction of the particles and be independent of grain size [9]. This is in contradiction with the different relaxation strengths in the single crystal and the polycrystalline sample (Fig. 6), both of which contained nearly identical volume fractions of particles. In addition, this mechanism is in conflict with the experimentally observed strong dependence of the maximum on prior deformation and recovery.

This leaves only a dislocation mechanism as an alternative explanation. Which dislocation mechanism can be reconciled with the experimental facts, in particular with the low activation enthalpy, can be narrowed down by model calculations. We therefore discuss the role of the solute atoms for the kinetics of dislocation movement. First it is shown that break-away of the dislocation from its solute cloud is unlikely. Consider a dislocation, which is pinned at distances $l$ and bows out under an external stress without taking the Cottrell cloud with it [26]. The resulting energy change $U$ comprises the work done by the external stress $\left(E_{\sigma}\right)$, the lengthening of the dislocation line $\left(E_{\mathrm{L}}\right)$, and the change in the interaction energy between the dislocation and the solute atoms $\left(\Delta E_{\mathrm{S}}\right)$

$$
U=\Delta E_{\mathrm{S}}+E_{\mathrm{L}}+E_{\sigma} .
$$

These terms are calculated as a function of the bowing-out distance $x_{\mathrm{d}}$ under similar assumptions as in Part I [14]:

(i) For the interaction energy between dislocation and solute atoms the elastic solution for a misfitting sphere in a hydrostatic stress field of a PeierlsNabarro edge dislocation $[14,27,28]$ is applied. The solute concentration around the dislocation at $x, y=0$ is calculated with the Fermi-Dirac distribution [29]. The change in the interaction energy $\Delta E_{\mathrm{S}}$ for a bowing-out distance $x_{\mathrm{d}}$ is given by the difference between the interaction energy for the bowed and the unbowed configuration.

(ii) To calculate the contribution from the lengthening of the dislocation line, the line tension is taken as $G b^{2} / 2$, where $G$ is the shear modulus and $b$ the Burgers vector. If the bowed-out configuration is described by a circular arc, the energy can be estimated from simple geometrical considerations as

$$
E_{\mathrm{L}}=\frac{4}{3} \cdot \frac{G \cdot b^{2}}{l} \cdot x_{\mathrm{d}}^{2} \text {. }
$$

(iii) The energy contribution due to the external stress is estimated as

$$
E_{\sigma}=-G \cdot \gamma \cdot b \cdot l \cdot x_{\mathrm{d}} / 2 \text {. }
$$

Table 3. Material data for Al-Si

\begin{tabular}{lcl}
\hline$\epsilon$ & -0.045 & {$[35]$} \\
$b(\mathrm{~nm})$ & 0.286 & {$[27]$} \\
$\xi(\mathrm{nm})$ & 0.1 & {$[27]$} \\
$G(\mathrm{GPa})$ & 26.5 & {$[27]$} \\
$v$ & 0.347 & {$[27]$} \\
$\Omega\left(\mathrm{m}^{3}\right)$ & $1.66 \times 10^{-29}$ & {$[27]$} \\
$D_{\mathrm{B}}\left(\mathrm{m} \cdot \mathrm{s}^{-2}\right)$ & $1.7 \times 10^{-4} \cdot \exp (-142000 / R T)$ & {$[39]$} \\
$D_{\mathrm{C}}\left(\mathrm{m} \cdot \mathrm{s}^{-2}\right)$ & $1.7 \times 10^{-4} \cdot \exp (-82000 / R T)$ & {$[39]$} \\
\hline
\end{tabular}

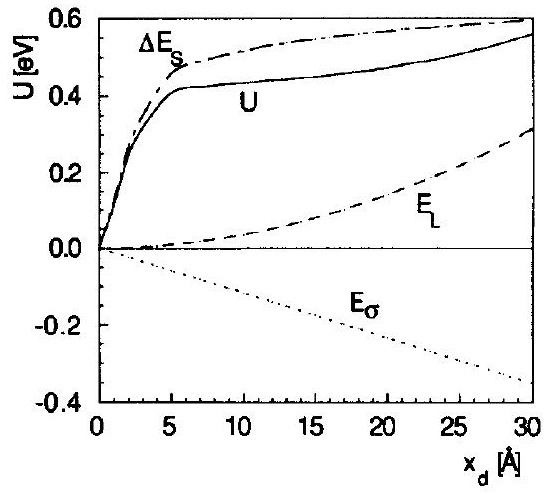

Fig. 11. The energy increment $U$ of a dislocation as function of the bowing-out distance $x_{\mathrm{d}}$, calculated under the assumption of a fixed solute cloud. $E_{\mathrm{L}}$ is the contribution from the line energy, $E_{\sigma}$ the external stress and $\Delta E_{\mathrm{s}}$ from the Cottrell cloud.

The calculations of $U$ in equation (2) were performed with the material data for Al-Si as listed in Table 3 and for $\sigma_{x y}=10^{-5} \cdot G, l=500 \mathrm{~nm}$, and $T=400 \mathrm{~K}$. As discussed in Appendix Al, a reasonable (lower) estimate for the solute concentration corresponding to the solubility limit of $\mathrm{Si}$ at $T=450 \mathrm{~K}$, is $c_{0} \approx 5$ $\times 10^{-5}$.

The results of these calculations are plotted in Fig. 11 as a function of $x_{d}$. It is seen that the energy $U$ of the bowed configuration is always higher than for the unbowed situation; the straight dislocation is, even under external stress, the stable configuration provided that the solute atoms do not move. Calculations with a variation of the parameter $c_{0}$ show that this statement holds for solute concentrations larger than $10^{-5}$. Thus, the dislocation can bow out only if the solute atoms diffuse with it and it is probable that this diffusion mechanism determines the kinetics of the dislocation movement. Such motion can be described by models of Winkler-Gniewek [30,31], Schoeck [13], and Seeger [32, 33].

Next we examine whether longitudinal movement of the solute, i.e. along the dislocation, or transverse movement, i.e. normal to the dislocation, is expected to dominate. The longitudinal movement of the solute near the dislocation may be favored as the measured activation enthalpy for this process is smaller than that for bulk diffusion; but for this effect to override the uniform distribution along the line, the equation

$$
\frac{\gamma^{2} \cdot G^{2} \cdot b^{2} \cdot l^{3}}{32 \cdot k T \cdot T_{\mathrm{L}}}>2.35 \cdot n
$$

must be fulfilled according to Winkler-Gniewek [30], where $T_{\mathrm{L}}$ is the line tension $\left(T_{\mathrm{L}} \sim G b^{2} / 2\right)$ and $n$ the number of solute atoms in the dislocation core of length $l$. The concentration in the dislocation core is calculated from

$$
c=c_{0} \cdot \exp \left(U_{\mathrm{B}} / k T\right)
$$

where $U_{\mathrm{B}}$ is the binding energy between the Si solute atoms and the dislocation, estimated as $0.2 \mathrm{eV}$ [14]. 
For $\sigma_{x y}=10^{-5} \cdot G, T=450 \mathrm{~K}, l=500 \mathrm{~nm}$ and the material data in Table 3 the left-hand side of equation (5) is an order of magnitude lower than the righthand side and longitudinal diffusion of the solute may thus be excluded.

\subsection{Comparison with our solute drag model [14]}

Following the arguments of the previous section, we can hypothesize on strong grounds that. under our effects visible in Fig. 6. For dislocation models of this type, the relaxation strength is always given by [13]

$$
\Delta \sim \frac{\rho \cdot l^{2}}{10}
$$

where $\rho$ is the dislocation density and $l$ the loop length between the pinning points. The increased internal friction after rolling (first run of SD in Fig. 6) may therefore be_ascribed to the higher dislocation experimental conditions, the Cottrell clouds move with the dislocations by a mechanism involving transverse diffusion. Several earlier models for such a mechanism exist, especially those of Schoek [13], which treats the dislocation as a string, and of Seeger $[32,33]$, who takes the kink structure of the dislocations in bcc metals into account. A discrepancy, however, which remains unsolved is the low measured activation enthalpy.

We have developed a model which considers the consequences of a higher solute mobility near the dislocation core [14]: At low solute concentrations $c_{0}$ and high strains $\gamma$, the kinetics of the dislocation motion is determined by fast diffusion of the solute atoms in the core, while for high $c_{0}$ and low $\gamma$ the diffusion of the solute far away from the dislocation is rate-limiting. Through calculations carried out for $\mathrm{Al}-\mathrm{Si}$, a map was constructed which delineates the parameter region where fast diffusion of the solute atoms in the dislocation core determines the relaxation process (Fig. 12). Also shown is the region investigated in our experiments, for which relaxation by solute diffusion in the core region ("core relaxation") is concluded to dominate.

Several predictions of this model are in encouraging agreement with our experimental results. First, the activation enthalpy calculated for this mechanism $\left(H_{\mathrm{m}}=1.02 \mathrm{eV}\right)$ corresponds reasonably well with the experiments $(H=1.16 \mathrm{eV}$, see Table 2$)$. [Differences in the inverse attempt frequency $\left(\tau_{0 \mathrm{~m}}=3.7 \times 10^{-17} \mathrm{~s}\right.$ and $\tau_{0 \mathrm{ex}}=2.9 \times 10^{-15} \mathrm{~s}$ ) should not be over-rated as the local processes in the dislocation core cannot be simulated with the model.]

Secondly, dislocation effects provide a natural explanation for the pre-straining and grain size

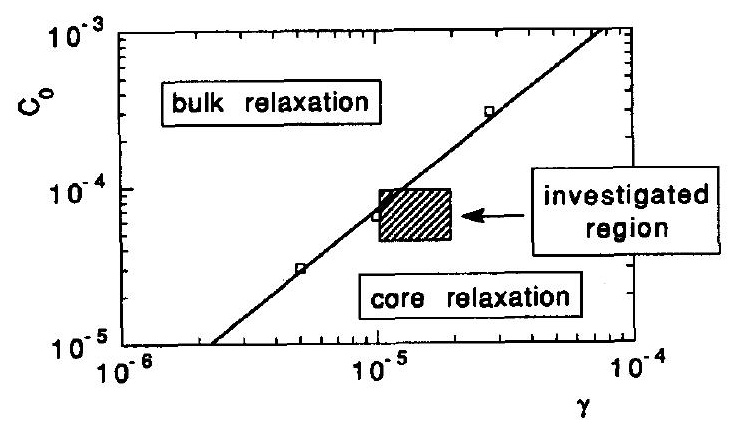

Fig. 12. Map for dominant internal friction processes as a function of the external strain $\gamma$ and the solute concentration $c_{0}$ for $\mathrm{Al}-\mathrm{Si}$ (from [14]). The experimentally investigated region is shown as the rectangle. density, whereas the lower internal friction in the second run reflects recovery during the first measurement. Also the higher relaxation strength in the polycrystalline sample compared to the single crystal S2 (Fig. 6) can be attributed to the higher dislocation density (compare Figs 1 and 5 for representative TEM images).

Further, for parameters where the solute in the dislocation core determines the kinetics an analytical expression for the relaxation time is given by equation (12) in [14]

$$
\tau=\frac{l^{2}}{5 \cdot G b^{2}} \cdot \frac{k T}{D} \cdot \frac{c_{0}}{b} \cdot \exp \left(U_{\mathrm{B}} / k T\right) .
$$

Because of the $l$-dependence, the relaxation time should be sensitive to the arrangement of the dislocations. The shift of the maximum to lower temperatures after deformation (specimen SD) may thus be explained by a shortening of the pinning length, possibly due to nodes produced by dislocation cutting processes.

Third, also the increasing relaxation strengths with increasing external stresses are in agreement with the model (Fig. 8 in [14]). Under a higher stress, the influence of the back stress exerted by the immobile solute atoms far away from the dislocation core is smaller and the dislocation can bow out more strongly.

Fourth, the activation volume may be estimated for the proposed mechanism as

$$
v_{\mathrm{m}}=\Delta l \cdot b^{2}
$$

where $\Delta l$ is the mean distance between the solute atoms in the dislocation core. For $T=450 \mathrm{~K}, U_{\mathrm{B}}$ $=0.2 \mathrm{eV}$ and $c_{0}=5 \times 10^{-5}$ we obtain $v_{\mathrm{m}}=116 \cdot b^{3}$, which is of the same order of magnitude as the experimental value $\left(v_{\mathrm{ex}}=180 \cdot b^{3}\right)$.

Finally, the dependence of the temperature at the maximum on the particle distance is a further test for the proposed mechanism. The condition for the maximum $\omega_{\mathrm{p}} \cdot \tau_{\mathrm{p}}=1$ gives, with equation (8)

$$
1=\omega_{\mathrm{p}} \cdot \tau_{\mathrm{p}} \sim \omega_{\mathrm{p}} \cdot T_{\mathrm{p}} \cdot l^{2} \cdot \exp \left(H / k T_{\mathrm{p}}\right) .
$$

(The subscripts $\mathrm{p}$ refer to values at the maximum.) Because the mean dislocation length between the pinning points is proportional to the mean planar particle distance, this equation can be written as

$$
\ln \left(\omega_{\mathrm{p}} \cdot T_{\mathrm{p}}\right)+H / k T_{\mathrm{p}}=-\mathrm{const}-2 \ln \left(l_{\mathrm{p}}\right)
$$

If the left hand side is plotted against $\ln \left(l_{p}\right)$, a linear relation with a slope of $n=2$ is expected. Such a 


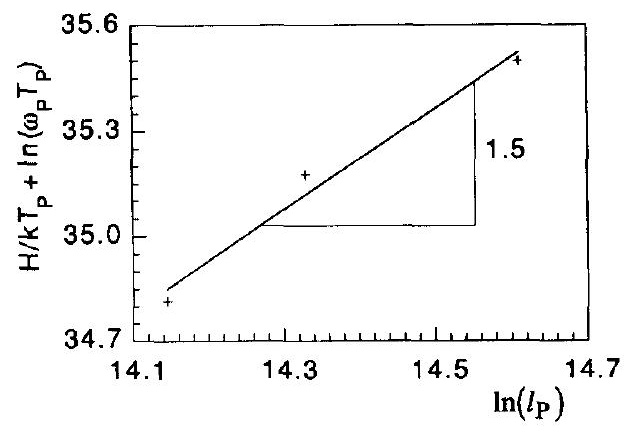

Fig. 13. Plot of equation (10) for our Al-Si data.

power law $\tau=l^{n}$ with $n=2$ also results from the models of Schoeck and Seeger. Such a plot (Fig. 13) gives, with the values extracted from Fig. 7, a reasonable fit for $n=1.5$.

In summary, the predictions of our solute drag model are in at least qualitative agreement with our experimental results on $\mathrm{Al}-\mathrm{Si}$. We therefore consider it as highly likely that the rate-controlling mechanism for internal friction in these alloys is based on the motion of solute atoms in the vicinity of the dislocation cores. To our knowledge, other models cannot provide an explanation for the low values measured for the activation enthalpy.

\subsection{Other experimental results}

We now attempt to reinterpret other experimental results from the literature in the light of our model. The discrepancy between the results of Okabe et al. [10] and Entwistle et al. [11] on nearly the same alloy as in the present work can probably be removed by the following arguments.

As the dislocation structure was not investigated by Okabe et al., only the dependence of the temperature at the maximum on the particle spacing can be compared with our model. Figure 14 shows the same plot as in Fig. 13 for these results, which gives a slope of $n=2.5$. The maximum observed for the single crystals may possibly be a result of accidental deformation during handling of the thinner and longer specimens in comparison to the samples in this investigation. The agreement with the model of Mori et al. [9] may well have been fortuitous. If on the other hand

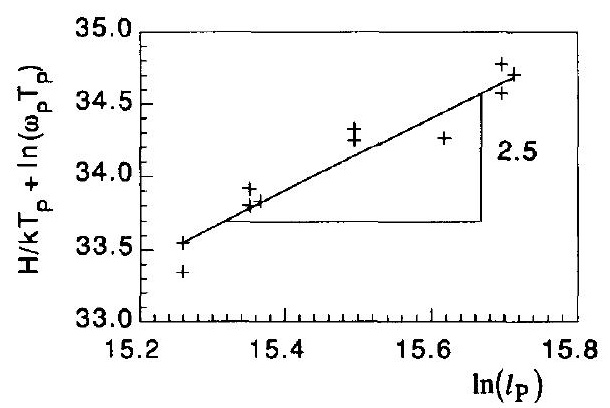

Fig. 14. Same plot as in Fig. 13 for the results of Okabe et al. [10]. the single-crystal specimens of Entwistle et al. were prepared and handled exceptionally well, it can be speculated that the lack of a pronounced dislocation structure produced the absence of the loss maximum in this case. It should be noted that for this comparison only specimens which had been aged by Entwistle et al. after quenching were included.

Also a loss maximum in rapidly solidified $\mathrm{Al}-\mathrm{Si}$ alloy [3] and an Al-Mg alloy [6] can be interpreted with the proposed dislocation model. In Al-0.96at.\% $\mathrm{Si}, \mathrm{Al}-3.8$ at. \% Si, and $\mathrm{Al}-11.5$ at. $\% \mathrm{Si}$ maxima were found between 400 and $500 \mathrm{~K}(f \sim 1 \mathrm{~Hz})$. The relaxation strength $\Delta$ and the temperature $T_{\mathrm{p}}$ at the maximum increased with higher aging temperatures, which may be attributed to Ostwald ripening of the particles and therefore longer dislocation segments [equations (7) and (8)]. The increase of $\Delta$ and $T_{\mathrm{p}}$ with decreasing $\mathrm{Si}$ content can be explained analogously.

A maximum in $\mathrm{Al}-\mathrm{Mg}(\mathrm{Al}-2.4 \mathrm{wt} \% \mathrm{Mg})$ [6] at $T \sim 550 \mathrm{~K}$ was attributed by the authors to a grain boundary mechanism. As the experiments were carried out only on polycrystalline samples and the activation enthalpy was not stated, a clear conclusion is not possible. It may be argued that because of the much higher solubility for $\mathrm{Mg}$ (than for $\mathrm{Si}$ ) and a higher elastic misfit $\left(\epsilon_{\mathrm{Mg}}=10.8 \%\right.$ [34], $\epsilon_{\mathrm{Si}}=-4.5 \%$ [35]), the bowing-out of the dislocation is likely to be determined by slow diffusion of the solute atoms far away from the dislocation (bulk diffusion). As the activation enthalpy for bulk diffusion of $\mathrm{Mg}$ in $\mathrm{Al}$ $(Q=120-130 \mathrm{~kJ}$ [36]) is higher than that for core diffusion of $\mathrm{Si}$ in $\mathrm{Al}$, the relaxation maximum would be shifted to a higher temperature, which is in agreement with the experimental result. The experimentally observed increase of the relaxation strength with longer aging times may again be a result of Ostwald ripening. Because of the lack of TEM results and single crystal data this interpretation must however remain somewhat speculative.

Finally, our model can explain why the solute content often has a strong effect on the shape of the internal friction spectra [37]. In a pure metal the activation enthalpy of the "grain boundary" maximum falls into the range for boundary self diffusion. For increasing solute concentration, an additional maximum often appears in the internal friction spectrum at a higher temperature, but at the expense of the original grain boundary maximum in the pure metal (Figs 15 and 16) [38]. For high concentrations only the high temperature maximum, whose activation enthalpy falls into the range for self diffusion in the bulk, is experimentally observed. This sequence in the internal friction spectra is in obvious agreement with the predictions of our dislocation model (see the schematically depicted spectra of Fig. 13 in [14]).

The critical concentration, at which the "cross-over" from the "low temperature" to the "high temperature" maximum occurs, can be calculated. On account of the small stacking fault energy in $\mathrm{Cu}$, the calculations were also conducted for the Burgers vector of a 


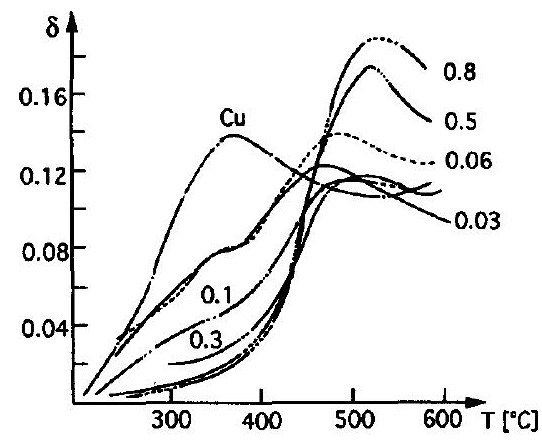

Fig. 15. The internal friction spectra for $\mathrm{Cu}$ with different Si content [38]. The "cross-over" concentration is about $10^{-3}$.

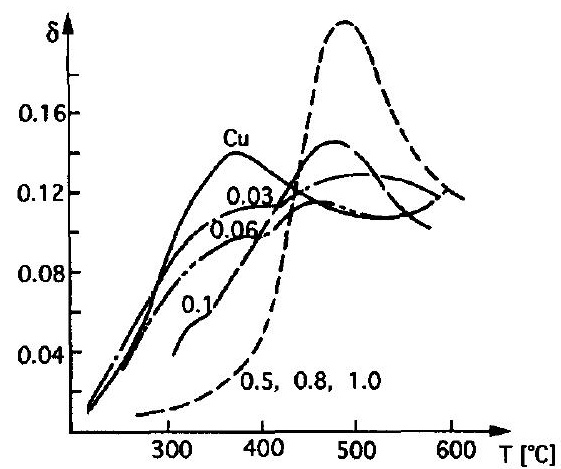

Fig. 16. The internal friction spectra for $\mathrm{Cu}$ with different Al content [38]. The "cross-over" concentration is about $3 \times 10^{-4}$.

partial dislocation. The calculation gives, for $T=$ $600 \mathrm{~K}, \gamma=5 \times 10^{-6}$ and the material data in Table 4, the values in Table 5 . It is encouraging that the calculated and measured values (Figs 15 and 16) are of the same order of magnitude.

Altogether, encouraging qualitative agreement could be obtained in several instances. It is however far more difficult to achieve full quantitative correspondence: critical material parameters such as the pinning length, the interaction energy between dislocation and solute atom and especially the concentration of

Table 4. Material parameter for $\mathrm{Cu}-\mathrm{Si}$ and $\mathrm{Cu}-\mathrm{Al}$

\begin{tabular}{lcccccc}
\hline & $G$ & $b$ & $b_{\mathrm{P}}$ & $\epsilon$ & $\epsilon$ & $\xi$ \\
$v$ & $(\mathrm{GPa})$ & $(\AA)$ & $(\AA)$ & $(\mathrm{Al}$ in $\mathrm{Cu})$ & $(\mathrm{Si}$ in $\mathrm{Cu})$ & $\begin{array}{c}\xi \\
(\AA)\end{array}$ \\
\hline 0.343 & 54.6 & 2.55 & 1.47 & $6.6 \%[40]$ & $1.8 \%[41]$ & 1.59 \\
\hline
\end{tabular}

Table 5. Calculated and experimental measured concentration for the transition from the core to the bulk relaxation

\begin{tabular}{lllc}
\hline Alloy & & $c_{\mathrm{th}}$ & $c_{\mathrm{ex}}$ \\
\hline $\mathrm{Cu}-\mathrm{Al}$ & $b$ & $4 \cdot 10^{-5}$ & $<3 \times 10^{-4}$ \\
& $b_{\mathrm{P}}$ & $8 \cdot 10^{-5}$ & \\
$\mathrm{Cu}-\mathrm{Si}$ & $b$ & $6 \cdot 10^{-4}$ & $(0.6-1) \times 10^{-3}$ \\
& $b_{\mathrm{p}}$ & $1 \cdot 10^{-3}$ & \\
\hline
\end{tabular}

(b-calculation with complete Burgers vector, $b_{\mathrm{p}}$-with Burgers vector of a Shockley partial). As a cross-over criterion a decrease of the relaxation strength for the pure grain boundary relaxation to $50 \%$ is used. To calculate the cross-over the approximation method described in [20] is applied. dissolved solute are not easily accessible. Nevertheless, the circumstantial evidence attests, in our view, to the plausibility of the assumption that internal friction may in several cases be controlled by core diffusion of dissolved solute atoms.

\section{SUMMARY}

The internal friction behavior of Al-Si alloys with incoherent $\mathrm{Si}$ particles was investigated. Experiments were conducted on a polycrystalline sample and on single crystals with different particle sizes and dislocation structures. In the alloys a loss maximum at $450 \mathrm{~K}$ for $f \sim 1 \mathrm{~Hz}$ was found with an activation enthalpy of $H=1.15 \pm 0.1 \mathrm{eV}$ and an inverse attempt frequency of $\tau_{0} \sim 3 \times 10^{-15} \mathrm{~s}$. From a consideration of both the microstructure, as investigated by TEM, and the mechanical loss spectra, internal friction mechanisms involving grain boundaries and stress relaxation in the particle were ruled out; the $\mathrm{Si}$ particles only stabilize the dislocation structure.

Model calculations suggested that the Cottrell clouds move with the dislocation by a mechanism involving transverse diffusion. As the measured activation enthalpy of the observed loss maximum was significantly smaller than that for bulk diffusion, our recent model for solute drag, which considers the higher mobility of the solute atoms in the core, was applied. The properties of the loss maximum, such as the amplitude dependence, the influence of the particle distance and of the microstructure, could be reasonably well explained with the proposed model.

The model was also applied to experiments reported in the literature on $\mathrm{Al}-\mathrm{Si}, \mathrm{Al}-\mathrm{Mg}, \mathrm{Cu}-\mathrm{Al}$, and $\mathrm{Cu}-\mathrm{Si}$, where it also seems to provide a natural explanation for the observed internal friction spectra. In conclusion, it is proposed that the internal friction behavior, even when characterized by a "low" activation enthalpy, need not be due to grain boundary or particle effects, but may be well explained by our model, which considers the effect of core diffusion on solute drag of dislocations.

\section{REFERENCES}

1. R. A. Winholtz and W. N. Weins, J. Physique 46, C10-375 (1985).

2. S. P. Wu, L. D. Zhang, G. Y. Tian and J. Z. Lu, Physica status solidi (a) 121, 149 (1990).

3. M. Tan and L. Liou, Mater. Sci. Engng A 114, 205 (1989).

4. T. Gongqing and H. Changzhi, Rare Metals 8, 20 (1989).

5. K. Zheng, S. Liu and D. Tian, Physica status solidi (a) 116, 621 (1989).

6. S. P. Wu and L. D. Zhang, Mater. Sci. Engng $A$ 132, 225 (1991).

7. T. Mori, M. Koda, R. Monzen and T. Mura, Acta metall. 31, 275 (1983).

8. S. E. Urretade Pereya and E. C. Moretti, Scripta metall. 23, 1691 (1989). 
9. T. Mori, M. Okabe and T. Mura, Acta metall. 28, 319 (1980).

10. M. Okabe. T. Mori and T. Mura. Phil. Mag. A 49.

(1981).

11. K. M. Entwistle, P. Fuller and I. Brough, Acta metall. 26, 1055 (1978).

12. G. Schoeck, Acta metall. 11, 617 (1963).

13. G. Schoeck, Scripta metall. 16, 233 (1982).

14. A. Pichler and E. Arzt, Acta metall. mater. 42, 3785 (1994).

15. R. A. Laudise, The Growth of Single Crystals, Solid State Physical Electronics Series, p. 113. Prentice-Hall, Englewood Cliffs, N.J. (1970).

16. L. J, Barker, Trans. Am. Soc. Metals 42, 347 (1949).

17. M. Weller and C. A. Wert, Stuttgarter Beiträge zur Naturkunde, $\mathrm{Cl} 8$ "Bernstein Neuigkeiten" (Herausg. Staatl. Museum für Naturkunde) (1984).

18. M. Weller. To be published.

19. A. S. Nowick and B. S. Berry, IBM J. Res. Develop. 5, 297, 312 (1961).

20. A. Pichler, Doctoral dissertation, Univ. Leoben and Max-Planck-Institut für Metallforschung, Stuttgart (1992).

21. T. S. Ké, Appl. Phys. 20, 274 (1949).

22. T. S. Ké, Scripta metall. 24, 347 (1990).

23. M. L. Nó, C. Esnouf, J. San Juan and G. Fantozzi, Acta metall. 36, 827, 837 (1988).

24. D. G. Blair, T. S. Hutchinson and D. H. Rogers, Can. J. Phys. 49, 633 (1971).

25. A. V. Granato and K. Lücke, J. appl. Phys, 52, 7136 (1981).

26. K. Lücke and J. Schlipf, in The Interaction Between Dislocations and Point Defects (edited by B. L. Eyre), Vol. 1, AERE Rep. 5944, p. 118 . Harwell (1968).

27. D. M. Barnett, G. Wong and W. D. Nix, Acta metall. 30, 2035 (1982)

28. J. P. Hirth and J. Lothe, Theory of Dislocations, 2nd edn, p. 218. Wiley, New York (1982).

29. P. N. Beshers, Acta metall. 6, 521 (1958).

30. W. Winkler-Gniewek, Doctoral dissertation, Aachen (1973).

31. W. Winkler-Gniewek, J. Schlipf and R. Schindlmayr, Proc. ICIFUAS 5 (edited by D. Lenz and K. Lücke), p. II-246. Springer, Berlin (1975).

32. A. Seeger, J. Physique 42, C5-201 (1981).

33. A. Seeger, Physica status solidi (a) 55, 457 (1979).

34. J. L. Murray, Bull. Alloy Phase Diagrams 3, 60 (1982).

35. J. L. Murray and A. J. McAlister, Bull. Alloy Phase Diagrams 5, 74 (1984).

36. Y. Minamino, T. Yamane, A. Shimomura, M. Shimada, M. Koizumi, N. Ogawa, J. Takahashi and H. Kimura, J. Mater. Sci. 18, 2679 (1983)

37. A. S. Nowick and B. S. Berry, Anelastic Relaxation in Crystalline Solids, p. 446. Academic Press, New York (1972).

38. S. Weinig and E. S. Machlin, Trans. Am. Inst. Min.

Engrs 209, 32 (1957).

39. H. J. Frost and M. F. Ashby, Deformation Mechanism Maps, p. 21. Pergamon Press, Oxford (1982).

40. A. J. Bradley and H. J. Goldschmidt, J. Inst. Metals 65 , 389 (1939).

41. A. G. H. Andersen (1940), quoted by R. W. Olesinski and G. J. Abbaschian, Bull. Alloy Phase Diagrams 7, 170 (1986)

\section{APPENDIX}

\section{A1. The Solute Atom Concentration}

For a comparison between the model and the experiments the concentration of dissolved solute atoms must be known In our calculation [14] a constant concentration in the bulk and an equilibrium segregation to the dislocation core were assumed. For a material with precipitates it is difficult to specify the right value of $c_{0}$. If the material would be in thermodynamic equilibrium the concentration as a function of the temperature may be written as

$$
c_{0}=c_{\mathrm{ph}} \exp \left(-T_{0} / T\right)
$$

which gives an additional contribution, $-R T_{0}$, to the measured activation enthalpy. As for an adjustment of the concentration to the equilibrium values large diffusion path $(\sim$ half particle distance $>100 \mathrm{~nm})$ are necessary in contrast to the relaxation where only a few jump are necessary, the assumption of a constant concentration, during the measurement may be convenient. This concentration distribution freezes in during the cooling of the samples. To get a measure for this freezing temperature and the concentration of solved atoms the mean diffusion path $\left[x=(D \cdot t)^{0.5}\right]$ for $1 \mathrm{~h}$ are calculated and listed for different temperatures in Table Al. According to this simple estimation a freezing temperature of $450-500 \mathrm{~K}$ and a concentration in the bulk of $c_{0} \sim 5 \times 10^{-5}-10^{-4}$ may be appropriate

For the local equilibrium around the dislocation only a few jumps are necessary and therefore a contribution from the binding energy to the measured activation enthalpy of the loss maxima, at least partially, may be determined.

Table Al. Mean diffusion path in the time $t=3600 \mathrm{~s}$ for different temperatures. The diffusion coefficient is the one for Al-Si selfdiffusion. Additionally, the solubility is listed [35]

\begin{tabular}{crr}
\hline$T(K)$ & $x(\AA)$ & \multicolumn{1}{c}{$c_{0}$} \\
\hline 450 & 44 & $3 \cdot 10^{-5}$ \\
500 & 300 & $1 \cdot 10^{-4}$ \\
550 & 14202 & $3.6 \cdot 10^{-4}$ \\
\hline
\end{tabular}

